\title{
Caracterización y distribución vertical de epífitas vasculares -orquídeas y bromelias- y hospederos en ecosistema de selva en sur de Perú
} Characterization and vertical distribution of
vascular epiphytes -orchids and bromeliads-
and hosts in rainforest ecosystem in southern Perú

Caracterização e distribuição vertical de epífitas vasculares -orquídeas e bromélias- e hospedeiros em um ecossistema florestal no sul do Peru

\author{
Hilber Ariosto Hurtado Alza1, Javier Orozco Ávila² \& Jhon Fredy Betancur Pérez \\ Ingeniero Forestal, Magister en Desarrollo Sostenible y Medio Ambiente; ${ }^{2}$ \\ Ingeniero Agrónomo, Magister en Producción vegetal. ${ }^{3}$ Licenciado en Biología y Química, \\ Especialista en Biología Molecular y Biotecnología, Doctor en Ciencias Agropecuarias.
}

1,2,3Universidad de Manizales. Manizales, Colombia

19argemiroforest@hotmail.com, 2javierorozcoavila@gmail.com, ${ }^{3 j}$ betancur@umanizales.edu.co

\section{Resumen}

Las epifitas son plantas que crecen principalmente adheridas a los troncos y ramas de árboles y arbustos. En la presente investigación se realizó el inventario de epífitas vasculares -orquídeas y bromelias- y sus hospederos, los cuales fueron caracterizados según su clasificación taxonómica; analizando también, el impacto sobre el componente de vegetación. Según los resultados, en las cuatro unidades de vegetación, se registraron 62 géneros, 308 especies epifitas y 15.520 individuos de epífitas. En relación con los forófitos se identificaron en total 266 especies de hospederos y 3.040 individuos arbóreos forófitos. En la familia Orchidaceae se identificaron 264 especies y 14.083 individuos; en la familia Bromeliaceae se identificaron 44 especies, agrupadas en 1.437 individuos. En el componente de vegetación el impacto fue negativo, sobre la vegetación arbórea u hospederos, ya que estos individuos serán talados, no obstante la vegetación epífita de orquídeas y bromelias serán trasladadas y monitoreadas, garantizando su sobrevivencia, caso que generará impacto positivo sobre este grupo de especies vegetales.

Palabras clave: epifitas vasculares, bromelias, orquídeas, hospederos.

\begin{abstract}
Epiphytes are plants that grow mainly adhered to trunks and branches of trees and shrubs. In this research, the inventory of epiphytes vascular-orchids and bromeliads-and their hosts, which were characterized according to their taxonomic classification, were carried out; Also analyzing the impact on the vegetation component. According to the results, in the four vegetation units, 62 genera, 308 epiphytes and 15,520 individuals were recorded. In relation
\end{abstract}


to the Forófitos were identified in total 266 species of hosts and 3,040 tree individuals forófitos. In the Orchidaceae family, 264 species and 14,083 individuals were identified; In the Bromeliaceae family, 44 species were identified, grouped in 1,437 individuals. In the vegetation component the impact was negative, on the tree or host vegetation, as these individuals will be felled, notwithstanding the epiphyte vegetation of orchids and bromeliads will be transferred and monitored, guaranteeing their survival, case that will generate positive impact on this group of plant species.

Key-words: vascular epiphytes, bromeliads, orchids, hosts.

\section{Resumo}

As epífitas são plantas que crescem principalmente ligadas aos troncos e ramos de árvores e arbustos. $\mathrm{Na}$ presente investigação, realizou-se o inventário de epífitas vasculares - oras e bromélias - e seus hospedeiros, caracterizados de acordo com sua classificação taxonômica; também analisando o impacto no componente da vegetação. De acordo com os resultados, nas quatro unidades vegetativas, 62 gêneros, 308 espécies epífitas e 15.520 indivíduos epífitos foram registrados. Em relação aos forófitos, foram identificados 266 espécies hospedeiras e 3.040 árvores forofíteas. Na família Orchidaceae, foram identificadas 264 espécies e 14.083 indivíduos; Na família Bromeliaceae, 44 espécies foram identificadas, agrupadas em 1.437 indivíduos. No componente da vegetação, o impacto foi negativo, na vegetação arbórea ou nos hospedeiros, já que esses indivíduos serão abatidos, porém a vegetação epífita de orquídeas e bromelias será transferida e monitorada, garantindo sua sobrevivência, no caso de gerar um impacto positivo nesse grupo de espécies de plantas.

Palavras chave: epífitas vasculares, bromélias, orquídeas, hospedeiros.

\section{Introducción}

El ecosistema selvático de la amazonia del Perú, se caracteriza por poseer árboles de gran tamaño, con un dosel cerrado, dentro del cual se pueden observar diferentes estratos. En un estrato es posible encontrar árboles de tamaños superiores a los 30 $\mathrm{m}$ de altura, árboles dentro del sotobosque, plantas rastreras y plantas que crecen alrededor o encima de otras plantas, como es el caso de las epifitas y los musgos (Acuña, 2012). En estos ecosistemas se encuentra una gran diversidad de especies tanto de flora como de fauna en total equilibrio. Dentro de una comunidad vegetal, las plantas epifitas juegan un papel importante desde el punto de vista ambiental, de acuerdo con su distribución en los diferentes estrados verticales de un ecosistema, sirven de hábitat y nicho para muchos organismos como hormigas, artrópodos, insectos y aves. Las epífitas solo usan a su hospedero como soporte o anclaje pues no toman ningún nutriente de éste para su desarrollo (Granados et al, 2003). Estudios realizados muestran que un ecosistema con presencia de plantas epifitas tiene más diversidad de una entomofauna asociada que otro ecosistema donde se han eliminado las epifitas. Algunas epifitas acumulan agua entre sus hojas generando una trama ecológica a su alrededor, mientras que otras pueden acumular cientos nutrientes importantes para sus hospederos. Para la captura de nutrientes los distintos grupos de epífitas presentan distintas estructuras y mecanismos, dependiendo de la fuente de suministro, ya que estos pueden provenir de la atmósfera, de la lixiviación de las hojas y corteza, de la acumulación de hojarasca y de la interacción con otros organismos, entre otros (Zotz \& Andrade, 2002).

En el año 2014 mediante Resolución Suprema No 054-2014-EM, se otorga a la Sociedad Concesionaria Gasoducto Sur Peruano S.A. la Concesión del Proyecto "Mejoras a la Seguridad Energética del País y Desarrollo del Gasoducto Sur Peruano". 
Esto hace necesario adelantar todos los estudios de impacto ambiental y buscar los mecanismos de reducir al máximo estos impactos, buscando formas de reemplazar o trasladar algunos especímenes de plantas a sitios aledaños a los intervenidos con las obras de infraestructura de exploración, extracción, conducción y utilización de éstas fuentes de energía. El objetivo de esta investigación fue caracterizar la población de epífitas vasculares -orquídeas y bromelias- y hospederos y analizar su distribución vertical, en un ecosistema de selva a intervenir con la construcción de la variante de conducción del gasoducto en el sur de Perú. El proyecto se realizó mediante el inventario al $100 \%$ de individuos arbóreos forofitos y los epífitos de las familias orchidiacea y bromeliaceae presentes en las tres zonas hipotéticas, definidas y modificas acorde a la división propuesta por Johansson (1974).

\section{Materiales y métodos}

\section{Área de estudio}

La investigación se realizó entre los KP 60+500m al KP 100+000m, tramo que se ubica en el área rural del centro poblado de Ivochote, que pertenece al distrito de Echarate, provincia de La Convención, región Cusco, Perú, en de la región natural de Selva y zona de vida de Selva Alta.

\section{Inventario de epífitas y sus hospederos}

El inventario de la población de especies epífitas y sus hospederos se llevó a cabo en un área total de 99 ha. Esta área está conformada por un derecho de vía (DdV) de $25 \mathrm{~m}$ de ancho y $39,5 \mathrm{~km}$ de longitud, de la construcción del gasoducto del sur del Perú. Se realizó un barrido en cada una de las unidades de vegetación, registrando los forófitos con diámetro a la altura del pecho igual o superior a $10 \mathrm{~cm}$, la información fue registrada en formatos de campo, donde se consigna información como la georefenciación, el nombre común y científico del forófito, y epífitos presentes en cada individuo arbóreo forófitos, teniendo en cuenta que en cada fórofito evaluado se divide en tres (3) zonas hipotéticas, que definen su distribución vertical: zona 1, parte basal del tronco, de 0 a $2 \mathrm{~m}$, zona 2, parte alta del tronco, a partir de los $2 \mathrm{~m}$ hasta la primera ramificación y zona 3 , el área de dosel o copa, definidas y modificadas de acuerdo con la división propuesta por Johansson (1974).

\section{Caracterización de la población de epífitas y sus hospederos}

Realizado el procesamiento de recolección de la información en campo, se determinó la composición florística de los epífitos y fórofitos u hospederos, para ello se analizó la diversidad de los epífitos, la riqueza está dada por el número de especies registradas de las dos familias estudiadas, Orchidacea y Bromeliacea, por unidad de cobertura, la diversidad se determina mediante los índices de ShannonWeiner y Simpson (Tabla 1).

Tabla 1. Índices de diversidad

\section{Índice}

Definición

Indice de diversidad de Shannon \& Wiener

Es igualmente una medida de la diversidad o riqueza en especies de una población dada
Dónde: $\mathrm{Pi}=a b u n d a n c i a$ proporcional de la especie i, lo cual implica obtener el número de individuos de la especie i dividido entre el número total de individuos de la muestra.

La ecuación de $D_{\mathrm{Si}}$ se aplica para comunidades 'finitas' donde todos los miembros han sido contados, es decir que $\mathrm{n}=\mathrm{N}$. ConsideÍndice de Simpson rando una comunidad 'extensa', un estimador adecuado de la diversidad calculado a partir de datos provenientes de una muestra de tamaño n sería:

$$
H^{\prime}=-\sum_{i=1}^{s}\left(p_{i} \times \log _{2} p_{i}\right)
$$

\section{Fórmula y/o Rangos de análisis}

$$
D^{\prime}{ }_{S i}=\sum_{i=1}^{S} \frac{n_{i}\left(n_{i}-1\right)}{n(n-1)}
$$




\section{Resultados y discusión}

\section{Caracterización de las unidades de vegetación} Al caracterizar la zona de influencia (99 ha), se identificaron cuatro unidades vegetativas, las cuales, se encuentran agrupadas ecológicamente como yungas orientales (YO), cada unidad presenta características paisajísticas particulares, que se encuentran influenciadas por su ubicación, altura, temperatura, actividad antrópica, (Tabla 2), el patrón de distribución espacial sobre los forófitos depende: de la especie del forófito, su posición, edad, condición y de la presencia de otras epifitas; según Sugden y Robins (1979), c.p Hernández, 2000, las epifitas son más abundantes en aquellos hospederos mus ramificados hacia todos los ángulos, con ramas horizontales y grandes copas.

Tabla 2. Unidades de vegetación caracterizadas en la zona de influencia.

\begin{tabular}{|c|c|c|}
\hline $\begin{array}{l}\text { Agrupación } \\
\text { ecológica }\end{array}$ & $\begin{array}{c}\text { Código } \\
\text { de unidad } \\
\text { de vegetación }\end{array}$ & $\begin{array}{c}\text { Unidad } \\
\text { de vegetación }\end{array}$ \\
\hline \multirow{4}{*}{$\begin{array}{l}\text { Yungas } \\
\text { orientales }\end{array}$} & Uv02-yo & $\begin{array}{l}\text { Bosque denso } \\
\text { montano y } \\
\text { basimontano }\end{array}$ \\
\hline & UV03-YO & $\begin{array}{l}\text { Bosque bajo, Arbustal, } \\
\text { Herbazal, densos } \\
\text { Basimontanos, en } \\
\text { Mesetas y Crestas }\end{array}$ \\
\hline & UV04-YO & $\begin{array}{l}\text { Bosque denso } \\
\text { Sub-Montano }\end{array}$ \\
\hline & UV07-YO & $\begin{array}{l}\text { Bosques y Matorrales } \\
\text { de densidad variada, } \\
\text { Sub-Montanos } \\
\text { y Basimontanos } \\
\text { secundarios }\end{array}$ \\
\hline
\end{tabular}

\section{Caracterización florística y diversidad}

\section{Composición florística de la población de especies epífitas}

En las cuatro unidades de vegetación se registraron 15.520 individuos representados en 308 especies, 62 géneros en las dos familias botánicas estudiadas, la Orchidaceae presenta 55 géneros, representados en 264 especies y 14,083 individuos y la Bromeliaceae con 7 géneros representados 44 especies en 1.437 individuos.

\section{Composición por género: familia Orchidaceae}

Las 264 especies de orquídeas identificadas en el área de estudio se agruparon en un total de 55 géneros diferentes, de los cuales 36 se ubicaron en la unidad de vegetación dos (UV02-YO) con 157 especies de orquídeas, el género Maxillaria, Epidendrum y Pleurothallis agruparon el mayor número de especies con un total de 20, 15 y 12 respectivamente; por su parte en la unidad vegetativa tres (UV03YO) se identificaron 35 géneros con 130 especies, destacándose el género Maxillaria con 17 especies, Pleurothallis con 14 y Epidendrum con 11 especies. En la unidad de vegetación cuatro (UV04-YO) se encontraron 44 géneros con 176 especies, de las cuales, el mayor número se agrupó en el género Maxillaria con 24 especies, 17 en el género Epidendrum y 14 en el género Pleurothallis, en cuanto a la unidad de vegetación siete (UV07-YO), se hallaron 29 géneros los cuales agruparon 89 especies de orquídeas, entre los de mayor abundancia de especies se encuentra el género Maxillaria con 13, y Pleurothallis y Epidendrum con 10 especies cada una (Figura 1). 

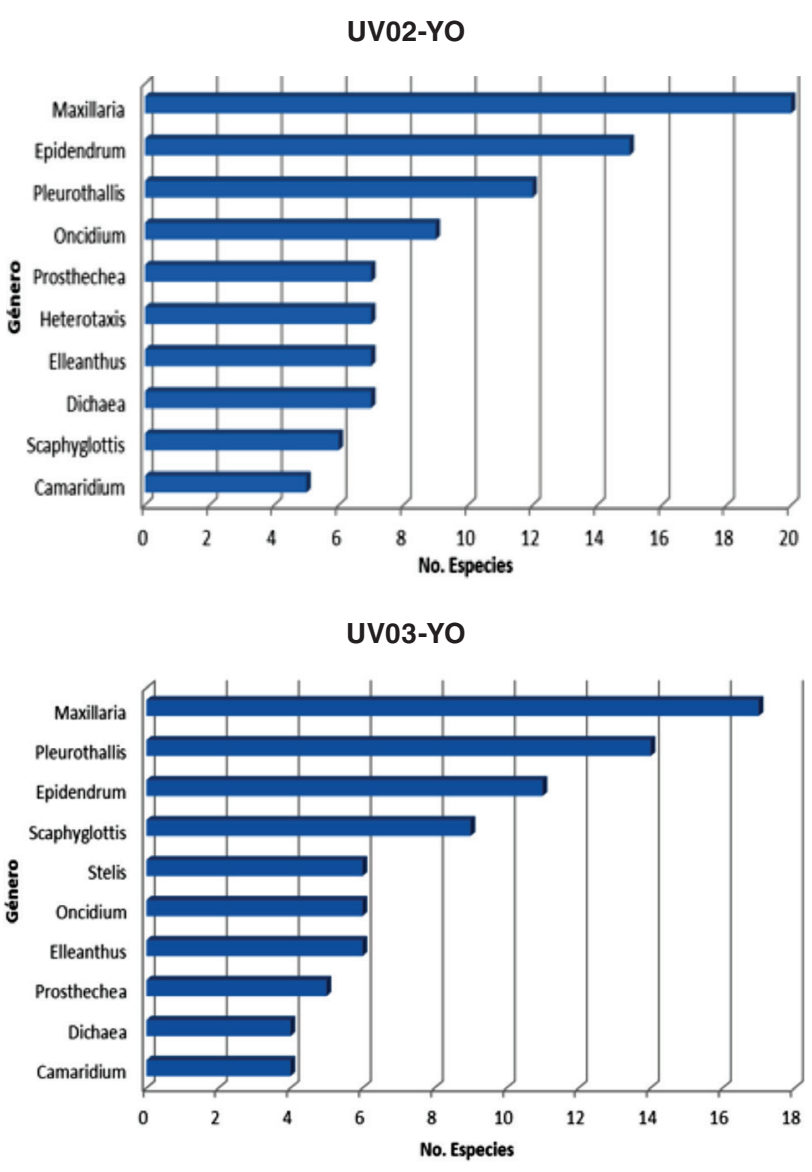

UV04-YO

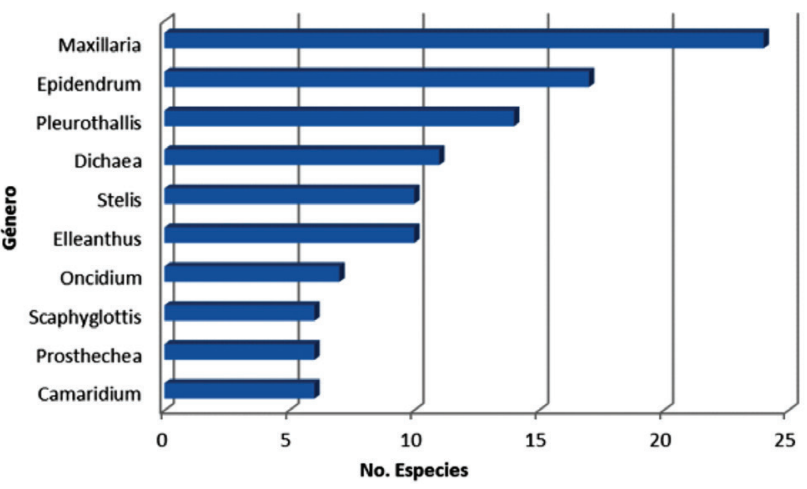

UV07-YO

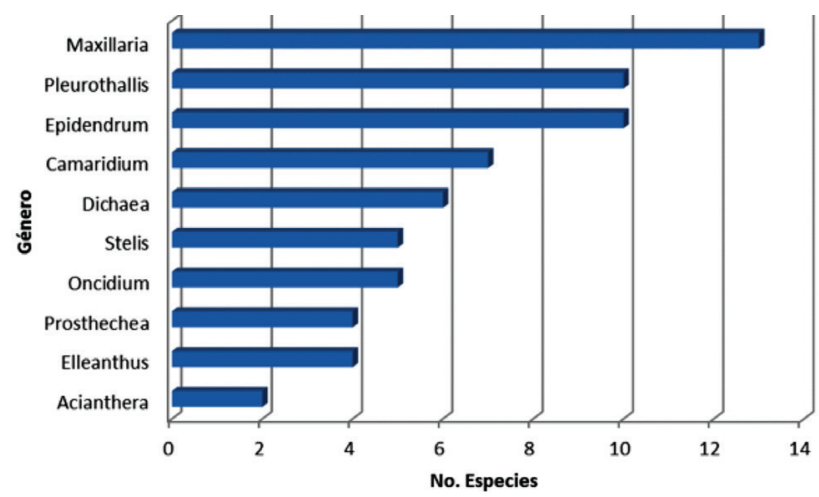

Figura 1. Composición por género: familia Orchidaceae

\section{Composición por género: familia Bromeliaceae}

La familia Bromeliaceae ha sido dividida en tres subfamilias bien definidas: Bromelioideae, Pitcairnioideae y Tillandsioideae (Smith y Downs, 1974 c.p Granados, 2005). En las cuatro unidades de vegetación se identificaron 7 géneros, los cuales agruparon en 44 especies de bromelias. En la unidad de vegetación (UV02-YO), se ubicaron cinco géneros con 30 especies diferentes, el género Guzmania albergó el mayor número de especies con un total de 12, seguido por Tillandsia con 9 y Aechmea con 5 especies. Por su parte, en la unidad de vegetación (UV03-YO), se encontraron cinco géneros con 21 especies, siendo el género Guzmania el de mayor participación con 9 especies, seguido por Tillandsia con 7 especies, los géneros Catopsis y Aechmea cuentan con 2 especies cada uno. En la unidad de vegetación (UV04-YO) se identificaron 6 géneros con 33 especies, de los cuales el género Tillandsia presentó el mayor número con un total de 13 , seguido por el género Guzmania con 11 y Aechmea con 5 especies de bromelias, Aguirre y Betancur (2008) hallaron tambien una amplia distribución de generos dentro de la familia Bromeliaceae, una de las caracteristicas que hace que el género Guzmania predomine sobre las otras especies son sus caracteristicas morfologicas, de acuerdo a Vargas (2002), quien describio al género por ser epífitas de bosques andinos, de tamaños variables hasta de un metro de altura, con las infloresencias cubiertas en formas de brácteas en espiral, son encontradas frecuentemente sobre árboles y arbustos a la vez que forman amplias colonias en el piso bajo del bosque, con brácteas de colores vistosos, caracteristica que hace que el género predomine, sobre otras especies de menor tamaño. Finalmente en la unidad (UV07YO) fue posible identificar 7 géneros con 23 especies, en donde los géneros Guzmania y Tillandsia se destacaron por albergar el mayor número con 10 y 7 especies respectivamente (Figura 2), se observó una amplia variabilidad y numero de especímenes de la familia bromeliaceae. Estas familias están incluidas dentro las familias reportadas para estudios en bosque premontanos y de selva baja (Huamantupa, 2010; Pitman, 2001; Gentry, 1988; Phillips et al., 1994), datos con una alta relación con los reportes realizados por Orozco et al. (2017). 

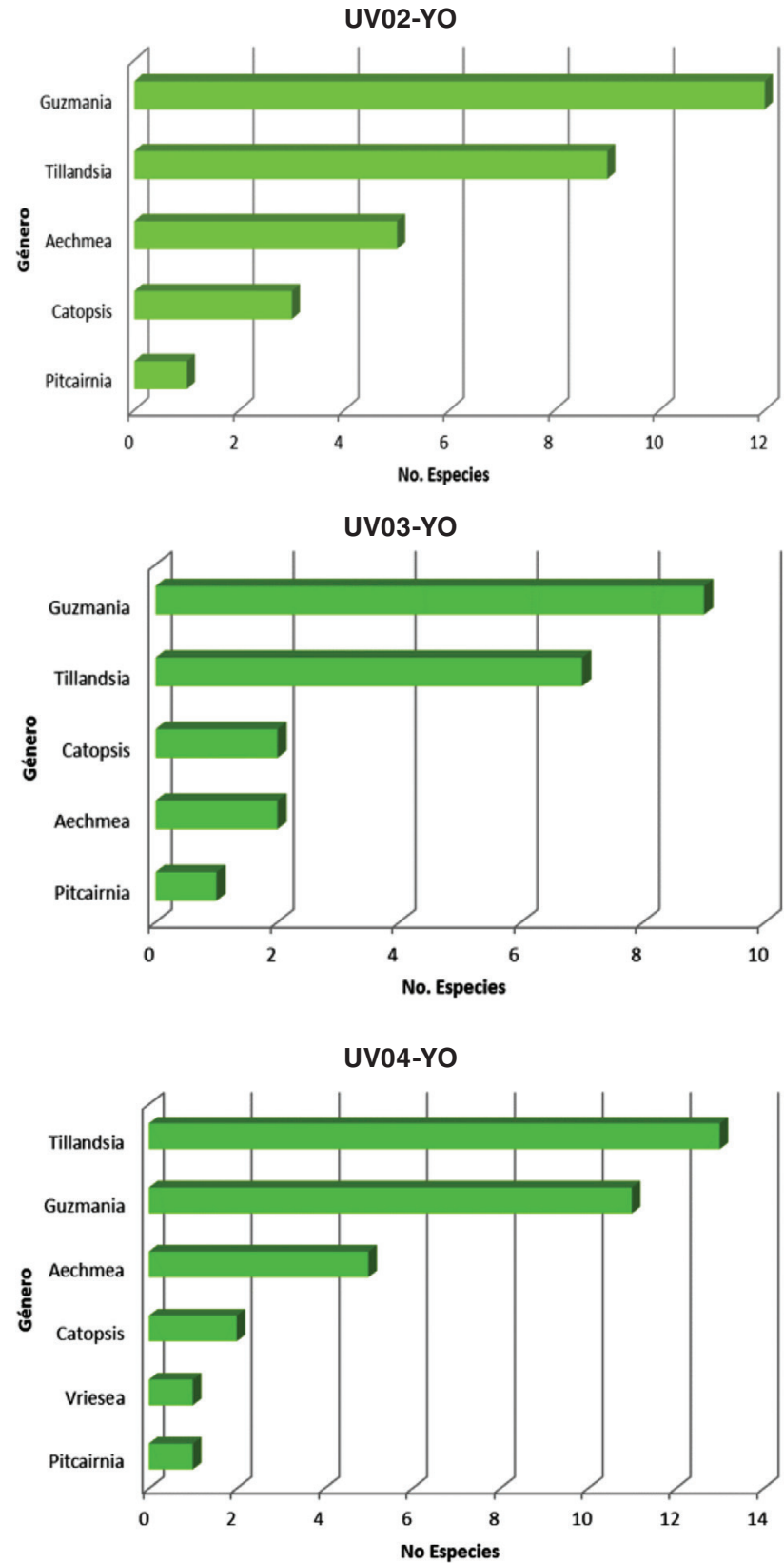

UV07-YO

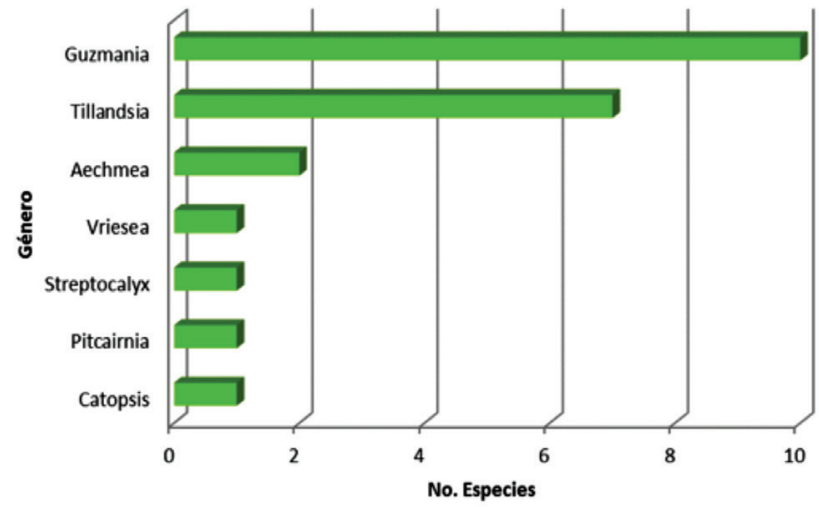

Figura 2. Composición por género: familia Bromeliaceae

\section{Composición florística de la población forofita y sus hospederos}

En las cuatro unidades de vegetación se registraron 3.040 individuos arbóreos representados en 266 especies, 171 géneros y 57 familias botánicas estudiadas. Las familias más representativas son Fabaceae con 34 especies, Moraceae con 26 especies, Rubiaceae con 17 especies y Euphorbiaceae con 16 especies, entre otras.

La unidad de vegetación (UV02-YO) conto con 981 individuos arbóreos forófitos, representados por 158 especies, 119 géneros y 51 familias, dentro de las cuales las más representativas son Fabaceae, Moraceae y Rubiaceae, con 18, 13 y 11 especies respectivamente; en la unidad vegetativa (UV03YO) se identificaron 605 individuos arbóreos forófitos, representados por 146 especies, 99 géneros y 33 familias, de las cuales, las más representativas son Fabaceae y Moraceae con 15 especies y Euphorbiaceae, con 11 especies. En la unidad de vegetación (UV04-YO) se encontraron 965 individuos arbóreos forófitos, representados por 167 especies, 116 géneros y 45 familias, siendo las más representativas Fabaceae, Moraceae y Urticaceae con 26, 18 y 11 especies respectivamente; en cuanto a la unidad de vegetación (UV07-YO), se hallaron 489 individuos arbóreos forófitos, representados por 60 especies, 51 géneros y 25 familias, siendo las más representativas Moraceae con 6 especies Urticaceae y Rubiaceae con 5 especies cada una ((Figura 3 ). 

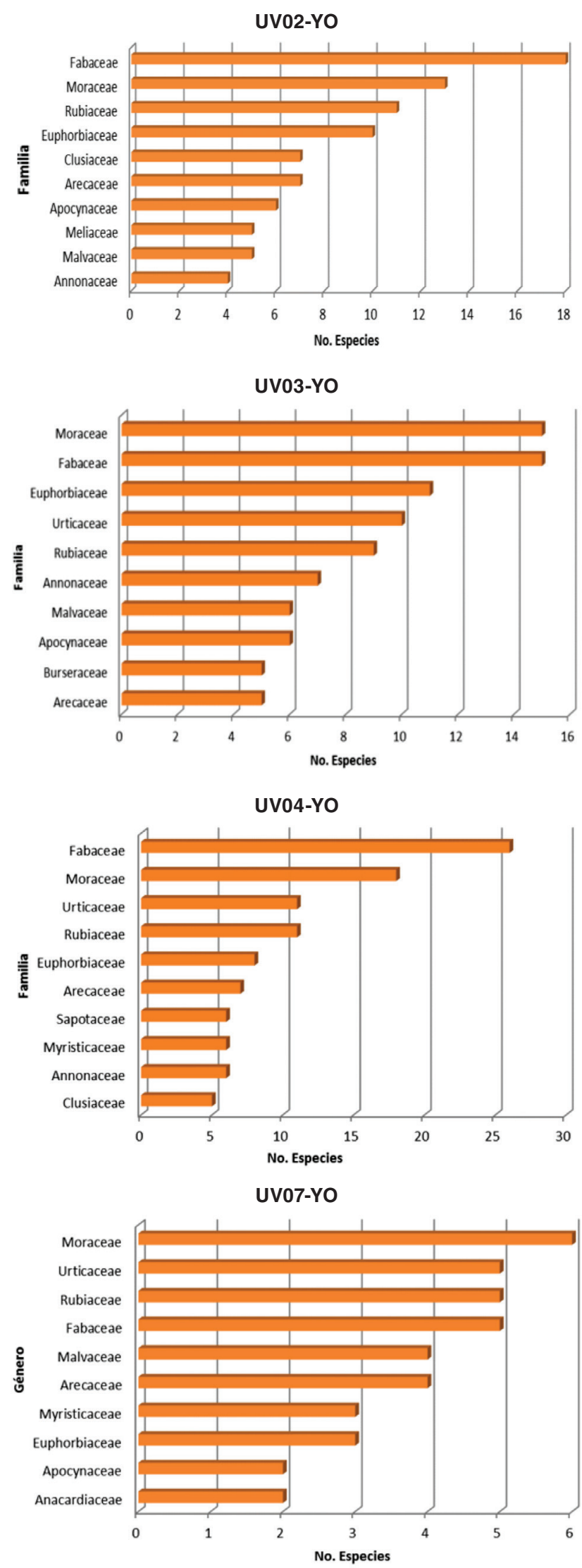

Figura 3. Composición florística de la población forofita u hospederos.
Las especies más abundantes en la unidad de vegetación (UV02-YO) son Clusia sp. (Tangariomanaki) perteneciente a la familia Clusiaceae con 48 individuos arbóreos forófitos, Miconia sp. (Oroengariki) y Graffenrieda sp.1 (Savotaroki 3), de la familia Melastomataceae, con 38 y 36 individuos arbóreos forófitos respectivamente. En la unidad de vegetación (UV03-YO) las especies más abundantes son Inga nobilis (Intsipa) de la familia Fabaceae con 48 individuos arbóreos forófitos, Blakea rosea (Savotaroki) de la familia Melastomataceae, con 33 individuos arbóreos forófitos y Himatanthus sucuuba (Chomisanto; Lagarto caspi) de la familia Apocynaceae, con 22 individuos arbóreos forófitos. Las especies más abundantes de la unidad de vegetación (UV04-YO) son Inga sp. 1 (Pacae) de la familia Fabaceae, Pseudolmedia laevis (Poshariki; Chimicua) de la familia Moraceae y Otoba glycycarpa (Tsonvantoki; Aguanillo) de la familia Myristicaceae, con 41, 40 y 37 individuos arbóreos forófitos respectivamente. Se ha onservado una amplia diversidad genetica y abundaciona de las epifitas, en los bosques mesófilos este tipo de plantas representa el $30 \%$ de la plantas vasculares (Viccon, 2009). En la unidad de vegetación (UV07-YO) las especies más abundantes son Wettinia augusta (Kepito) de la familia Arecaceae, Inga nobilis (Intsipa) de la familia Fabaceae y Ocotea javitensis (Inshoviki) de la familia Lauraceae, con 72, 42 y 41 individuos arbóreos forófitos respectivamente, la amplia diversidad genética encontrada está relacionada con los datos encontrados en el inventario realizado por Arévalo (2004), en el oriente de Colombia donde encontró 2.015 epífitas vasculares, pertenecientes a 183 especies, 71 géneros y 27 familias.

\section{Diversidad de epifitas}

La diversidad se compone de dos elementos, variedad o riqueza y abundancia relativa de especies, su expresión se logra mediante el registro del número de especies, la descripción de la abundancia relativa o mediante el uso de una medida que combine los dos componentes (Magurran, 2013). La riqueza y diversidad se determinó a nivel de unidades de vegetación, siendo la UV07-YO la que presenta mayor riqueza, y esto puede estar dado a que es la unidad 
con mayor área, seguida de la unidad UV02-YO, el índice de Shannon-Weiner $\left(\mathrm{H}^{\prime}\right)$ mide la heterogeneidad y el valor máximo (5), los resultado muestra una muy alta diversidad para las unidades de vegetación UV02-YO, UV03-YO y UV07-YO, la unidad UV07-YO muestra una diversidad media-alta, lo que puede indicar una intervención antrópica marcada en esta unidad. El índice de Simpson $\left(D_{\mathrm{Si}}\right)$ es casi similar en las cuatro unidades de vegetación, estando muy cerca a uno, lo que evidencia que no hay una dominancia resaltada de alguna o algunas especies de epífitos en las unidades estudiadas, teniendo en cuenta que cuando el valor del índice se acerca más a uno menor es la dominancia, por lo cual los ecosistemas estudiados tiene muy alta diversidad, esto puede deberse a que existen las riquezas son altas y las especies con mayor abundancia son pocas, situación que impide a las especies más abundantes dominar significativamente en el ecosistema (Tabla 3).

Tabla 3. Diversidad de epífitos por unidad de vegetación.

\begin{tabular}{l|c|c|c}
\hline $\begin{array}{c}\text { Unidades } \\
\text { de Vegetación }\end{array}$ & Riqueza & Índice de Shannon-Weiner (H') & Índice de Simpson $\left(\mathbf{D}_{\mathrm{si}}\right)$ \\
\hline UV02-YO & 187 & 4,298 & 0,961 \\
\hline UV03-YO & 131 & 4,579 & 0,960 \\
\hline UV04-YO & 209 & 3,291 & 0,977 \\
\hline UV07-YO & 112 & 4,691 & 0,954 \\
\hline
\end{tabular}

\section{Análisis de especies epífitas y hospederos}

La unidad de vegetación que presenta un mayor número de especies epífitos es la unidad UV04-YO, representada por 50 géneros y 4.035 individuos, estos se ubican en 965 forófitos, de 167 especies; seguidamente por la unidad UV02-YO, cuenta con 187 especies epífitos, representados por 41 géneros y 5.571 individuos, ubicados en 981 forófitos pertenecientes a 158 especies; la unidad UV03-YO cuenta con 151 especies epífitos, constituidos por 40 géneros y 3.690 individuos, ubicados en 605 forófitos pertenecientes a 146 especies y por último la unidad UV07-YO presenta 112 especies epífitos, representados por 36 géneros y 2.224 individuos, ubicados en 489 forófitos pertenecientes a 60 especies. Los forófitos hospedan orquídeas, en otros casos solo bromelias y otros comparten las dos especies (Tabla 4).

Tabla 4. Especies de epifitos (Orquídeas y Bromelias) y forófitos en las cuatro unidades de vegetación.

\begin{tabular}{l|c|c|c|c|c}
\hline $\begin{array}{c}\text { Unidad } \\
\text { de vegetación }\end{array}$ & Género & No. Especies & No. Individuos & $\begin{array}{c}\text { No. Especies } \\
\text { forófitos }\end{array}$ & $\begin{array}{c}\text { No. individuos } \\
\text { forófitos }\end{array}$ \\
\hline UV02-YO & 41 & 187 & 5571 & 158 & 981 \\
\hline UV03-YO & 40 & 151 & 3690 & 146 & 605 \\
\hline UV04-YO & 50 & 209 & 4035 & 167 & 965 \\
\hline UV07-YO & 36 & 112 & 2224 & 60 & 489 \\
\hline TOTALES & & 659 & 15520 & - & 3040 \\
\hline
\end{tabular}


El mayor número de especies de orquídeas se halló en la unidad de vegetación cuatro (UV04YO) con 176 especies y un total de 3.576 individuos, cabe recordar que ésta unidad es la de mayor área evaluada con 37.97 ha, aportando así el $66.67 \%$ de la totalidad de especies de orquídeas encontradas. Seguidamente en la unidad de vegetación dos (UV02-YO) se identificaron 157 especies de orquídeas con 5.293 individuos, representando el $59.47 \%$ de las especies de orquídeas encontradas en el área de estudiada; por su parte en la unidad vegetativa tres (UV03-YO) fue posible localizar 130 especies para un total de 3.458 individuos con un $49.24 \%$; finalmente en la unidad vegetativa siete (UV-07-YO) se localizaron 89 especies con 1.756 individuos participando con un $33.71 \%$ sobre la totalidad de especie de orquídeas encontradas (Tabla 5).

Tabla 5. Especies de orquídeas epifitas y hospederos en las cuatro unidades de vegetación.

\begin{tabular}{l|c|c|c|c|c}
\hline $\begin{array}{c}\text { Unidad } \\
\text { de vegetación }\end{array}$ & Género & No. Especies & No. Individuos & $\begin{array}{c}\text { No. Especies } \\
\text { forófitos }\end{array}$ & $\begin{array}{c}\text { No. individuos } \\
\text { forófitos }\end{array}$ \\
\hline UV02-YO & 36 & 157 & 5293 & 148 & 850 \\
\hline UV03-YO & 35 & 130 & 3458 & 138 & 496 \\
\hline UV04-YO & 44 & 176 & 3576 & 158 & 769 \\
\hline UV07-YO & 29 & 89 & 1756 & 53 & 312 \\
\hline TOTALES & & & 14083 & - & 2427 \\
\hline
\end{tabular}

Las unidades con mayor número de especies bromelias fueron la UV04-YO y UV02-YO, cada una con 33 y 30 especies respectivamente, sin embargo el número de individuos fue variable, en la unidad UV02YO se hallaron 278 individuos, entre tanto la unidad
UV04-Y estuvo conformada por 459 individuos. En la unidad de vegetación UV03-YO se identificaron 21 especies de bromelias con 232 individuos, entre tanto en la unidad UV07-YO se encontraron 23 especies representadas en 468 individuos (Tabla 6).

Tabla 6. Especies de bromelias epifitas y hospederos en las cuatro unidades de vegetación.

\begin{tabular}{l|c|c|c|c|c}
\hline $\begin{array}{c}\text { Unidad } \\
\text { de vegetación }\end{array}$ & Género & No. Especies & No. Individuos & $\begin{array}{c}\text { No. Especies } \\
\text { forófitos }\end{array}$ & $\begin{array}{c}\text { No. individuos } \\
\text { forófitos }\end{array}$ \\
\hline UV02-YO & 5 & 30 & 278 & 71 & 177 \\
\hline UV03-YO & 5 & 21 & 232 & 59 & 134 \\
\hline UV04-YO & 6 & 33 & 459 & 84 & 241 \\
\hline UV07-YO & 7 & 23 & 468 & 42 & 203 \\
\hline TOTAL & & & 1437 & - & 755 \\
\hline
\end{tabular}

Análisis de la población de hospederos en relación a la preferencia de las epífitas -Orquídeas y Bromelias.

\section{Número de hospederos por unidad} de vegetación: Familia Orchidaceae

En las cuatro unidades de vegetación se identificaron
259 especies de hospederos y 2427 individuos arbóreos forófitos, con presencia de las especies de la familia Orchidaceae. La amplia diversidad genética de la familia Orchidaceae, ha sido identificada en otras regiones, tal como lo reporta Wolf y Flamenco (2003) en el estado de Chiapas (México) donde realizo el estudio en un bosque mesófilo con una 
altitud de entre los 500 y 2000 msnm, encontrando 1.173 especies de epífitas, de las cuales la mitad fueron orquídeas, seguidas por bromelias y helechos. En la unidad de vegetación dos (UV02-YO) se hallaron 5.293 individuos de orquídeas epífitas distribuidas en 850 forófitos los cuales se representan en 178 especies, los de epífitos que presentaron mayor colonización de individuos de forófitos son: Inti bicallosa (Rchb.f.) M.A.Blanco con 61 individuos se encuentra colonizando en 48 forofitos, seguida por Stelis sp.1 y Scaphyglottis prolifera (R.Br.) Cogn., con 684 y 65 individuos respectivamente, colonizando 42 fórofitos cada una, entre otras. En la unidad de vegetación tres (UV03-YO) se registraron 3.458 individuos de orquídeas, los cuales se encontraron colonizando 496 forófitos agrupados en 138 especies diferentes, la especie Stelis sp.1 colonizó el mayor número de hospederos con un total de 30 individuos arbóreos y 460 individuos de epífitas, seguida por la especie Stelis sp. 2 con 21 hospederos colonizados por 331 individuos de epífitas de ésta especie. En la unidad de vegetación cuatro (UV04-YO) se encontraron 3.576 individuos de orquídeas colonizando 769 forófitos representados en 158 especies, la especie con mayor número de forófitos colonizados fue Pleurothallis revoluta (Ruiz \& Pav.) Garay, con un total de 56 individuos arbóreos y 87 individuos de epífitas; seguida de la especie Pleurothallis ruscifolia (Jacq.) R.Br. tuvo una colonización de 25 individuos arbóreos por 33 individuos de epífitas. En la unidad de vegetación siete (UV07-YO) se identificaron en total 312 hospederos agrupados en 53 especies, colonizados por 1.756 individuos de epífitas, la especie Epidendrum sp.1 colonizó en total 23 hospederos con 38 individuos de epífitas; por su parte en las especies Pleurothallis revoluta (Ruiz \& Pav.) Garay y Camaridium vestitum (Sw.) Lindl., fueron reportados en 20 y 17 forófitos colonizados por 87 y 24 individuos de epífitas respectivamente (Figura 4); la familia Orchidaceae constituye para la flora peruana la familia más diversa, con alrededor de 212 géneros y 2020 especies (León, Pitman \& Roque, 2006), aunque se estima que el número real podría oscilar entre 2500 y 3500 especies (Collantes, 2003), la mayoría son hierbas epífitas o terrestres, aunque hay también las que presentan ambos hábitos.
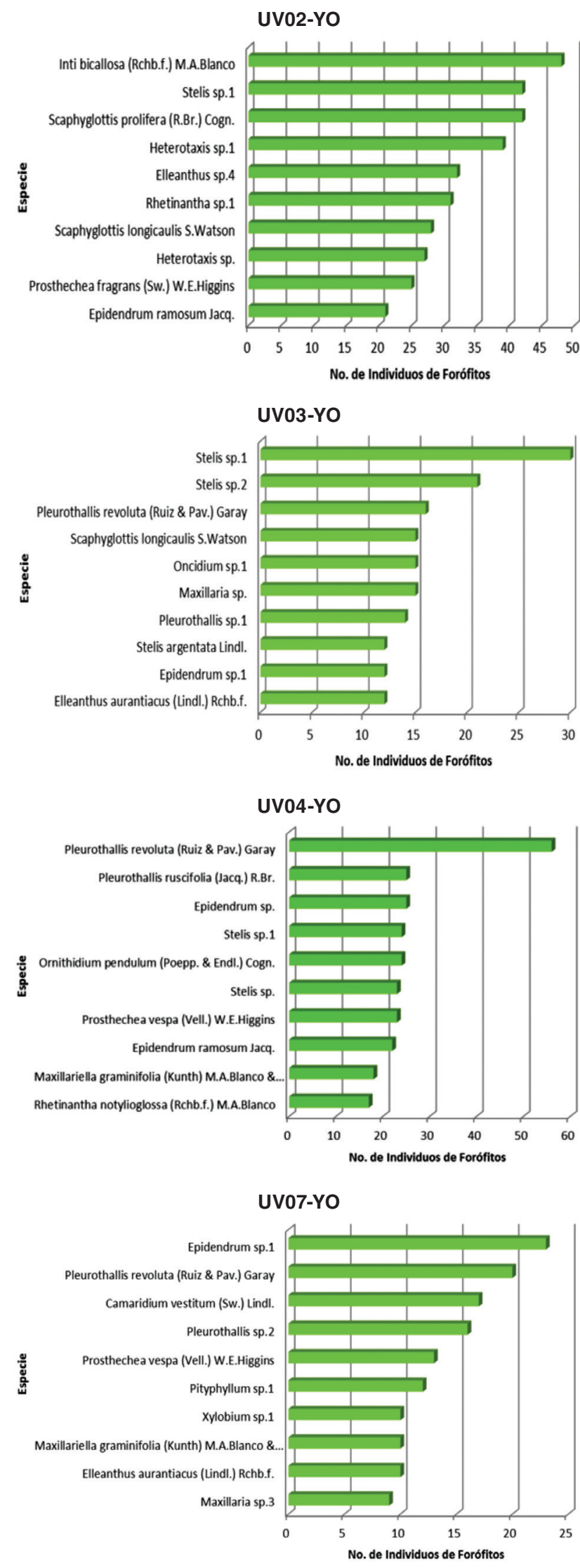

Figura 4. Número de hospederos por unidad de vegetación: Familia Orchidaceae. 


\section{Número de hospederos por unidad}

\section{de vegetación: Familia Bromeliaceae}

En las cuatro unidades de vegetación se encontraron 152 especies de hospederos y 755 forófitos, colonizados por especies de la familia Bromeliaceae. En la unidad de vegetación dos (UV02-YO) se registraron 177 hospederos de 71 especies y colonizados por 278 especies de bromelias agrupadas en 30 especies, la especie Guzmania jaramilloi H.E.Luther se registró con 78 bromelias epífitas colonizando 61 hospederos; seguidamente la especie Tillandsia sp.4 fue registrada con 30 individuos de epífitas coIonizando 20 forófitos. En la unidad de vegetación tres (UV03-YO) se encontraron 134 forófitos de en 59 especies, colonizados por 232 bromelias epífitas e en 21 especies, Guzmania melinonis Regel con 36 hospederos colonizados por 55 bromelias epífitas; seguidamente se encontró la especie Tillandsia sp.2 con 23 individuos de epífitas colonizando 17 forófitos. En la unidad de vegetación cuatro (UV04YO) se registraron 241 forófitos de 84 especies, los cuales fueron colonizados por 459 bromelias epifitas agrupadas en 33 especies, la especie Guzmania lingulata (L.) Mez presentó 52 hospederos colonizados por 65 bromelias epifitas, seguida por Guzmania melinonis Regel colonizó 42 hospederos con 57 individuos de epifitas. En La unidad de vegetación siete (UV07-YO) se identificaron 203 individuos arbóres hospederos, de en 42 especies, colonizados por 23 especies de bromelias para un total de 468 individuos, Entre las especies de mayor colonización se encuentran Guzmania melinonis Regel con 59 individuos arbóreos forófitos colonizados por 83 bromelias epífitas, Catopsis sp.1 con 46 individuos arbóreos forófitos colonizados por 67 bromelias epifitas (Figura 5). En las bromeliáceas epífitas han evolucionado una gran diversidad de estructuras de carácter adaptativo y mecanismos fisiológicos (formación de tanques para la captación de agua, suculencia foliar, tricomas foliares especializados en la absorción de agua y nutrimentos, así como la fotosíntesis CAM), que les han permitido adaptarse con éxito al ambiente epífito caracterizado por pulsos cortos de agua, altas intensidades lumínicas e inestabilidad de las ramas de los forófitos ante los vientos fuertes (Benzing, 1990, 1998).
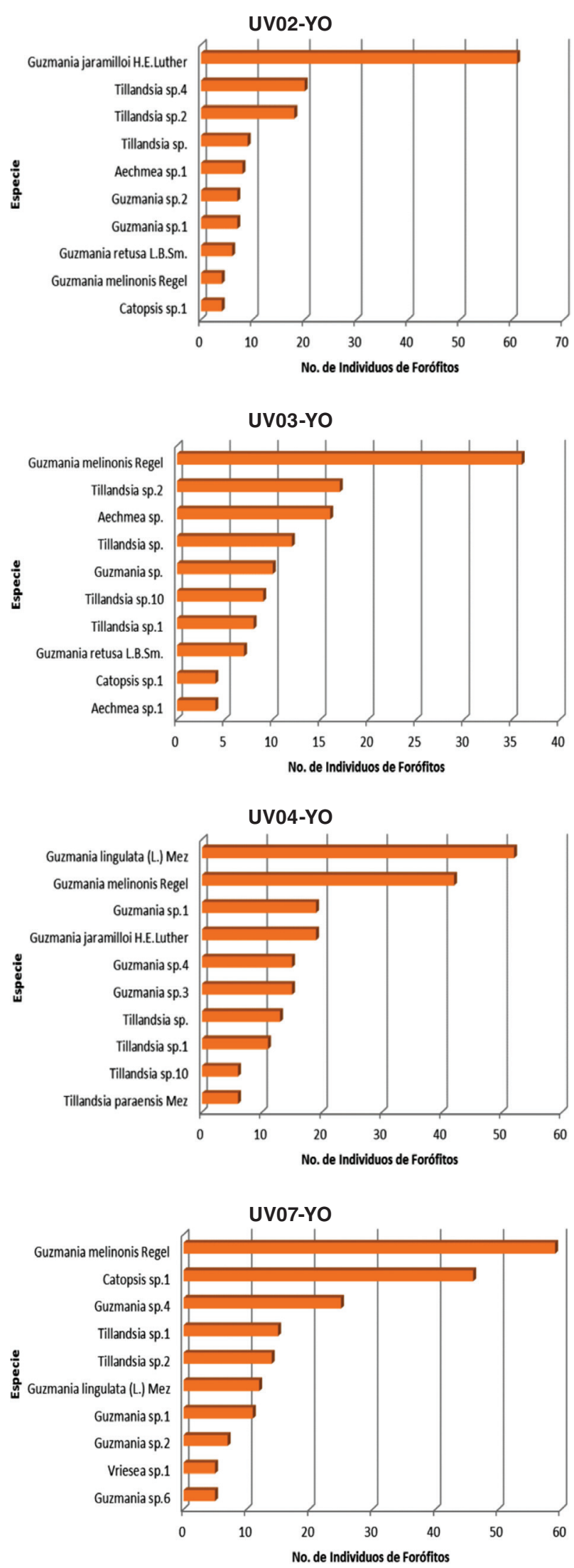

Figura 5. Número de hospederos por unidad de vegetación: Familia Bromeliaceae. 
Distribución de vertical: Número de individuos de epífitos por estratos del forófito

De acuerdo con la información registrada en las cuatro unidades de vegetación, para el caso de las orquídeas epifitas, el $58.06 \%$ de los individuos se ubicaron en el estrato dos de los individuos arbóreos hospederos, las cuales están más adaptadas a una intensidad lumínica somera, por su parte el $21.27 \%$ de las epifitas colonizaron el estrato uno, las cuales se encuentran adaptadas a una intensidad lumínica muy baja y el $20.67 \%$ se ubicó en el estrato tres, las cuales se adaptan a una intensidad lumínica alta (Figura 6); Según Hernández (2000), la distribución de las epifitas vasculares a varios niveles dentro del bosque, parece ser el resultado de un balance entre los requerimientos de luz y suministro de agua, debido al gradiente vertical de estos elementos dentro del bosque.

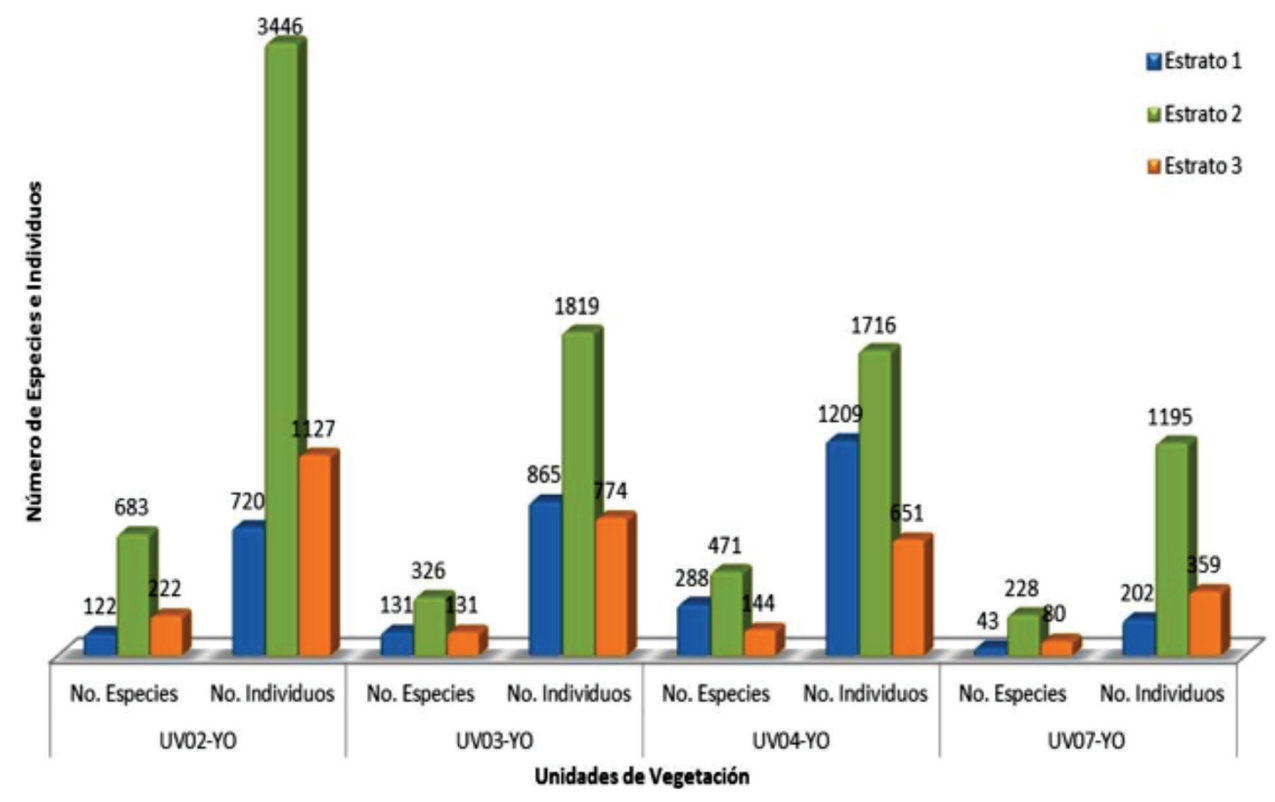

Figura 6. Distribución vertical de especies de Orquídeas epifitas en el hospedero o forófito.

La distribución de las bromelias epífitas en las cuatro unidades de vegetación, fue mayor en el estrato dos con $41.96 \%$ de ubicación en ésta área del árbol, por su parte el $38.69 \%$ de los individuos se distribuyeron en el primer estrato y el $19.35 \%$ en el tercer estrato (Figura 7). La distribución de las plantas se rige por las condiciones climáticas favorables para ellas; es así como, la temperatura influye sobre los rangos metabólicos, la luz condiciona la fotosíntesis, y ambas influyen sobre la apertura estomática que permite la transpiración, de acuerdo a déficits de vapor de agua atmosféricos y del grosor de la capa límite foliar (Benzing, 1998; Lambers, Chapin \& Pons 1998, Cach-Pérez, Andrade \& Reyes-García 2014). Por lo tanto, si existen cambios en las condiciones climáticas en general, podría esperarse, como consecuencia, que las especies sigan una de tres alternativas: adaptarse, migrar o extinguirse, lo cual dependerá, en gran medida, de la rapidez con los que se presenten los cambios en el clima (Dawson et al., 2011, Cach et al, 2014). 


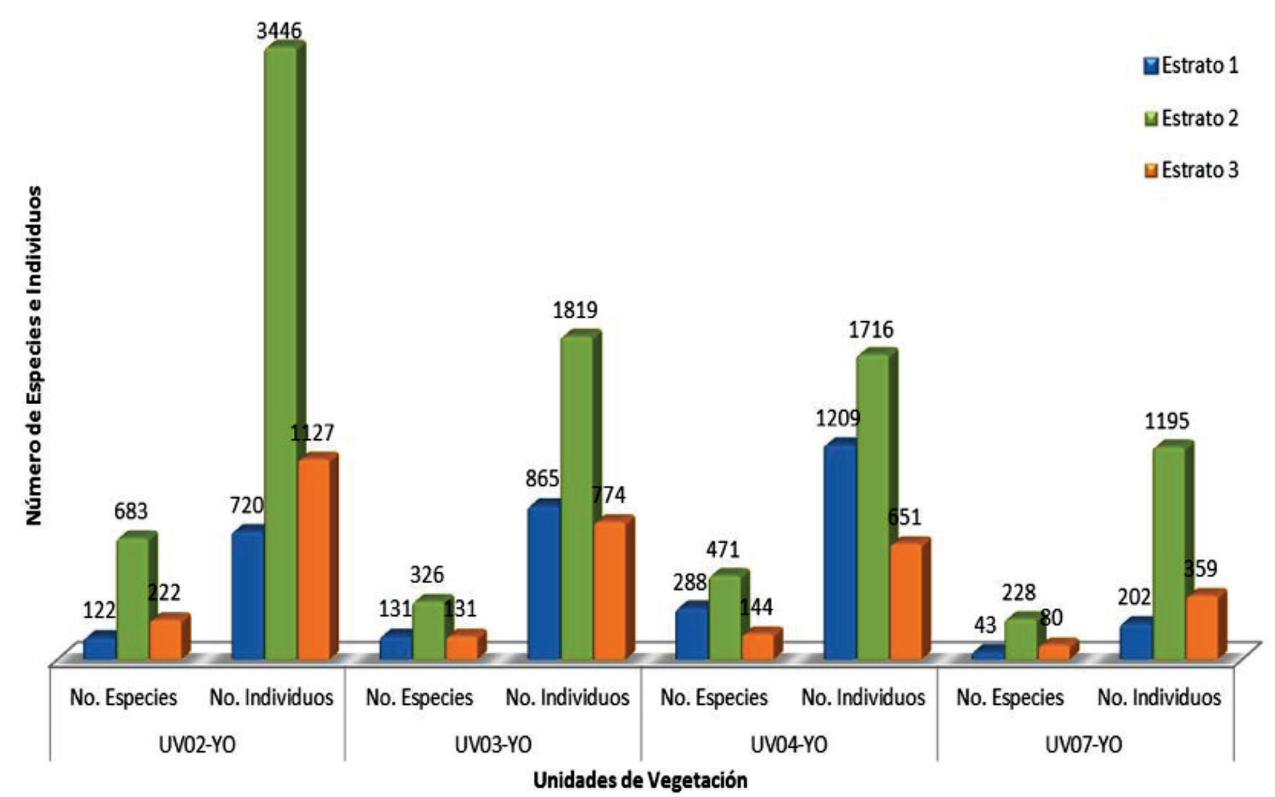

Figura 7. Distribución vertical de especies de Orquídeas epifitas en el hospedero o forófito.

\section{Impacto ambiental sobre la vegetación}

El mayor impacto ambiental sobre la vegetación se presenta en la etapa de trabajo preliminar y construcción, ya que se necesitará ejecutar actividades de desbroce y tala para la liberación del derecho de vía (DdV) donde se instalará el gasoducto, así como las instalaciones auxiliares de apoyo para la ejecución del proyecto (campamentos, centros de acopio de tuberías y caminos de acceso, Zona de Disposición de Material Excedente (ZODME).

Los componentes del Proyecto y las instalaciones auxiliares afectarán vegetación de tipo Herbazal arbolado, Bosque y arbustal submontano y basimontano, Matorral submontano. En estas unidades de vegetación se pueden registrar las siguientes especies: Melinis minutiflora (Familia Poaceae), Axonopus elegantulus (Familia Poaceae) y Paspalum humboldtianum (Poaceae); mientras que entre los arbustos se tiene a la Vernonia sp1 (Familia Asteraceae) y Baccharis chilco (Familia Asteraceae), porsnetadas en la unidad de herbazal arbolado. Las especies de una mayor presencia son de uso forestal como Ocotea sp1 y 2 (Familia Lauraceae) y Nectandra sp2 (Familia Lauraceae), ubicado en la unidad de Bosque y arbustal submontano y basimontano y por último en la unidad matorreeal submoentamos podemos encontrar especies reportadas localmente como de uso maderable (Ocotea sp1) entre las herbáceas destacan especies como Melinis minutiflora (Familia Poaceae) y Cenchrus bambusiformis (Familia Poaceae).

\section{Conclusiones y recomendaciones}

En las cuatro unidades de vegetación en donde se realizó el inventario, las familias más representativas de los forófitos u hospederos fueron Fabaceae, Moraceae, Rubiaceae y Euphorbiaceae, etc.; el inventario en la zona de influencia reveló que dentro de las dos familias botánicas estudiadas Orchidaceae y Bromeliaceae; se registraron 15.520 individuos de epífitas representados por 62 géneros y 308 especies epifitas; se identificaron 264 especies correspondientes a la familia Orchidaceae, para un total de 14.083 individuos; en relación con la familia Bromeliaceae se identificaron 44 especies, agrupadas en 1.437 individuos. La familia con mayor representatividad en las cuatro unidades de vegetación, es Orchidaceae con 55 géneros, 264 especies y 14.083 individuos; los géneros más representativos para la familia Orchidaceae fueron Maxillaria, Epidendrum y Pleurothallis; siendo el género Maxiliraia 
el que alberga el mayor número de especies diferentes de orquídeas (33). Entre las especies identificadas con mayor representatividad se encontraron Maxillaria xylobiiflora Schltr.; Pleurothallis ruscifolia (Jacq.) R.Br. y Epidendrum purum Lindl. El 61.6\% de las especies registradas en el inventario para la familia Orchidaceae se encuentra sin identificar taxonómicamente a nivel de especie; respecto a la riqueza y diversidad por unidad de vegetación, se evidenció que en las cuatro unidades cuentan con una gran riqueza y muy alta diversidad, pero sobresale el Bosque denso sub-montano (UV04-YO), con la riqueza y la diversidad más alta y los Bosques, seguido del Bosque denso Montano y Basimontano (UV02-YO).

El mayor número de especies de orquídeas se halló en la unidad de vegetación cuatro (UV04-YO) con 176 especies y un total de 3.576 individuos, de igual manera registra el mayor número de especies de hospederos con un total de 158 pertenecientes a 769 individuos arbóreos forófitos. La unidad de vegetación dos (UV02-YO) registró el mayor número de orquídeas epifitas con un total 5.293 individuos. igualmente en esta unidad se reportó el mayor número de individuos arbóreos forófitos con 850 individuos agrupados en 148 especies; de estos se registraron especies de las familias Melastomataceae, Poaceae y Urticaceae, las cuales son especies indicadoras de la actividad antrópica.

La familia Bromeliaceae estuvo representada por 7 géneros, 44 especies y 1.437 individuos. Las unidades dos y la cuatro registraron el mayor número de especies bromelias, cada una con 30 y 33 especies, sin embargo el número de individuos fue variable, en la unidad dos se hallaron 278 individuos y en la unidad cuatro 459 individuos. En la unidad siete se registraron el mayor número de bromelias epifitas con un total de 468 individuos agrupados en 23 especies. La distribución vertical de las bromelias epífitas en las cuatro unidades de vegetación, fue mayor en el estrato dos con $41.96 \%$ de ubicación en ésta área del árbol, por su parte el $38.69 \%$ de los individuos se distribuyeron en el primer estrato $y$ el $19.35 \%$ en el tercero; los géneros con mayor representatividad dentro de la familia Bromeliaceae fueron Tillandsia, Guzmania y Aechmea. El género Tillandsia agrupó en las cuatro unidades de vegetación el mayor número de especies con un total de 17. Entre las especies identificadas con mayor representatividad se encontraron Tillandsia parviflora Ruiz \& Pav.; Guzmania melinonis Regel y Aechmea zebrina L.B.Sm. El $65.9 \%$ de las especies de bromelias registradas en las cuatro unidades de vegetación aún no cuentan con una clasificación taxonómica a nivel de especie.

Existe en el área de influencia del estudio un mayor número de géneros y especies de la familia orquidiaceae, así como un mayor número de individuos, que en la familia bromeliaceae. Los datos registrados en el inventario tanto para orquídeas como para bromelias, se convierten en un posible indicador de la riqueza en biodiversidad que alberga ésta zona del Perú, pero también de la ausencia de investigación orientada a la taxonomía de especies y su posterior reporte como especies nuevas.

El mayor impacto ambiental negativo se presenta sobre la vegetación arbórea la cual debe ser eliminada en la fase de apertura del derecho de vía, la población epífita presenta un impacto positivo dado que previo a las actividades de tala se realiza el rescate y posterior reubicación en ecosistemas similares a lado y lado del derecho de vía a una distancia prudencial, a las cuales se les realizara monitoreo y mantenimiento, con el fin de garantizar la sobrevivencia de un mínimo del $90 \%$ de los epífitos trasladados; en el componente de vegetación el impacto es negativo, de influencia a lo largo del DdV, directo, de intensidad baja a media, acumulativo, sinérgico, temporal, reversible, mitigable, lo que determina un nivel de importancia alta. Una consecuencia grave sobre el ecosistema intervenido es la pérdida que podría llegar a darse sobre el germoplasma en ésta área del país, según los resultados más del $50 \%$ de la especies de epífitas no están identificadas taxonómicamente.

Es necesario realizar estudios orientados a la taxonomía de especies, teniendo en cuenta que en ésta investigación fue posible evidenciar el alto porcentaje de especies sin identificar, y las cuales podrían 
perderse si al momento del traslado a otro hospedero no presentan una respuesta positiva.

Es conveniente realizar un impacto ambiental de las zonas aledañas (desde el borde de la obra hasta lo profundo del ecosistema) a la obra de infraestructura, ya que una vez realizada la misma todas las condiciones del ecosistema podrían cambiar en relación con los factores climáticos y bióticos. Es importante tener en cuenta las condiciones del ecosistema en las zonas aledañas donde van a ser trasladadas las especies epífitas, ya que, las condiciones climáticas y bióticas cambiarían con el paso de la obra y es necesario garantizar la sobrevivencia de las especies.

Es preciso realizar investigaciones que profundicen en aspectos fisiológicos y ecológicos de las epifitas, ya que estas especies son altamente susceptibles al cambio del clima; los cuales, servirán de orientación para estudios de cambio climático y servicios ambientales que ofrecen los ecosistemas y de ésta manera generar conocimiento necesario para el desarrollo de estrategias de conservación.

\section{Literatura citada}

1. Acuña Tarazona, M. E. (2012). Flora epífita vascular representativa de bosque montano y de llanura amazónica del Parque Nacional Yanachaga Chemillén (Oxapampa, Pasco).

2. Aguirre, S. \& Betancur, J. (2008). Sinopsis del género Aechmea (Bromeliaceae) para Colombia. Caldasia, 30, (2) 265-288. Recuperado de: http://www.scielo. org.co/pdf/cal/v30n2/v30n2a2.pdf.

3. Arévalo, R. (2004). Diversidad de epífitas vasculares en cuatro bosques del sector suroriental de la serranía de Chiriboquete, Guayana - Colombiana. Caldasia 26(2),359-380.

4. Benzing, D. (1990). Vascular epiphytes. General biology and related biota. Cambridge University Press. New York. 354p.

5. Benzing, D. (1998). Vulnerabilities of tropical forests to climate change: the significance of residents epiphytes. Climatic change, 39, (2-3), 519-540.

6. Cach-Pérez, M. J., Andrade, J. L. \& Reyes-García, C. (2014). La susceptibilidad de las bromeliáceas epífitas al cambio climático. Botanical Sciences, 92(2), 157-168.
7. Collantes, B. (2003) Epiifitas del Peru. En: Rumbos Perú. No. 13. 6p. Recuperado de: http://www.rumbosperu.com

8. Dawson, T. P., Jackson, S. T., House, J. I., Prentice, I. C. \& Mace, G. M. (2011). Beyond predictions: biodiversity conservation in a changing climate. Science, 332(6025), 53-58.

9. Granados-Sánchez, D., López-Ríos, G. F., HernándezGarcía, M. Á., \& Sánchez-González, A. (2003). Ecología de las plantas epífitas. Revista Chapingo. Serie ciencias forestales y del ambiente, 9(2).

10. Gentry, A. H. (1988). Changes in plant community diversity and floristic composition on environmental and geographical gradients. Annals of the Missouri Botanical Garden, 1-34.

11. Huamantupa-Chuquimaco, I. (2010). Inusual riqueza, composición y estructura arbórea en el bosque de tierra firme del Pongo Qoñec, Sur Oriente peruano. Revista Peruana de Biología, 17(2), 167-171.

12. Hernández-Rosas, J. I. (2000). Patrones de distribución de las epifitas vasculares y arquitectura de los forofitos de un bosque húmedo tropical del Alto Orinoco, Edo. Amazonas, Venezuela. Acta Biol. Venez, 20(3), 41-58.

13. Johansson, D. (1974). Ecology of vascular epiphytes in west African rain forest. Acta Phytogeograpica Suecica. 59: 1-136.

14. Lambers, H., Chapin III, F. S. \& Pons, T. L. (1998). Photosynthesis, respiration, and long-distance transport. In Plant physiological ecology (pp. 10-153). Springer New York.

15. León, B., Pitman, N. \& Roque, J. (2006). Introducción a las plantas endémicas del Perú. Revista peruana de biolog ía, 13(2), 9-22.

16. Magurran, A. E. (2013). Ecological diversity and its measurement. Springer Science \& Business Media.

17. Orozco, A., Valencia, M. \& Betancur, P. (2017). Estimation of the transfer of vascular epiphytes, as a conservation strategy in the municipality of Aguazul, Casanare, Colombia. Revista de Investigación Agraria y Ambiental (RIAA) Vol. 8, Núm. 1. https://doi. org/10.22490/issn.2145-6453.

18. Phillips, O. L., Hall, P., Gentry, A. H., Sawyer, S. A. \& Vasquez, R. (1994). Dynamics and species richness of tropical rain forests. Proceedings of the National Academy of Sciences, 91(7), 2805-2809.

19. Pitman, N. C., Terborgh, J. W., Silman, M. R., Núñez, V., Neill, D. A., Cerón, C. E. \& Aulestia, M. (2001). Dominance and distribution of tree species in upper Amazonian terra firme forests. Ecology, 82(8), 2101-2117.

20. Pla, L. (2006). Biodiversidad: inferencia basada en el índice de shannon y la riqueza Interciencia, 31, (8), 583-590.

21. Smith, L. B. \& Downs, R. J. (1974). Flora neotropica monograph no. 14. (Pitcairnioideae) (Bromeliaceae).

22. Sugden, A. M. \& Robins, R. J. (1979). Aspects of the ecology of vascular epiphytes in Colombian cloud forests, I. The distribution of the epiphytic flora. Biotropica, 173-188. 
23. Vargas, W. G. (2002). Guía ilustrada de las plantas de las montañas del Quindío y los Andes Centrales. Universidad de Caldas.

24. Viccon Esquivel, J. (2009). Riqueza y composición florística de las epífitas vasculares del Bosque Mesófilo de Montaña de las localidades de Atzalán y Zongolica, Veracruz (Doctoral dissertation).
25. Wolf, J. H. \& Alejandro, F. S. (2003). Patterns in species richness and distribution of vascular epiphytes in Chiapas, Mexico. Journal of Biogeography, 30(11), 1689-1707.

26. Zotz, G. \& Andrade, J. L. (2002). La ecología y la fisiología de las epífitas y las hemiepífitas. Ecología y conservación de bosques neotropicales, 271-296. 